\title{
Cross Entropy Method for Solving Generalized Orienteering Problem
}

\author{
Budi Santosa, Nur Hardiansyah \\ Indusrial Engineering, Institut Teknologi Sepuluh Nopember Surabaya, Surabaya, Indonesia. \\ E-mail: budi_s@ie.its.ac.id
}

Received August 16 ${ }^{\text {th }}, 2010$; revised October 11 ${ }^{\text {th }}, 2010$; accepted November $27^{\text {th }}, 2010$.

\begin{abstract}
Optimization technique has been growing rapidly throughout the years. It is caused by the growing complexity of problems that require a relatively long time to solve using exact optimization approach. One of complex problems that is hard to solve using the exact method is Generalized Orienteering Problem (GOP), a combinatorial problem including NP-hard problem. Recently, there has been plenty of heuristic method development to solve this problem. This research is an implementation of cross entropy $(C E)$ method in real case of GOP. CE is an optimization technique that relatively new, using two main procedures; generating sample solution and parameter updating to produce better sample for next iteration. At this research, GOP problem that occurs at finding optimal route consist of 27 cities in eastern China is investigated. Results indicate that CE method give better performance than those of Artificial Neural Network (ANN) and Harmony Search $(H S)$.
\end{abstract}

Keywords: Generalized Orienteering Problem, Cross Entropy, Combinatorial Problem

\section{Introduction}

Transportation model is a classic problem that still interesting to be discussed. One of transportation problem is the Traveling Salesman Problem (TSP). TSP is a problem where a salesman must visit a set of cities, where each city must not be visited more than once, and the finishing point is the same as the starting point. The result of this problem is a tour that gave the shortest tour distance. One of the TSP problems is Multi Objective Vending Problem (MVP), which is not a necessary for a salesman to visit each city. One of MVP is the Orienteering Problem (OP). This problem is a combinatorial problem that including in NP-Hard Problem.

Orienteering Problem is one type of TSP with score. Score is a benefit or a satisfaction that gained from visiting the node (city). The objective of an OP is to define the tour that front end the same node, that maximize total score without break over the maximum distance constraint. This problem inspired by outdoor sport that usually played at mountain or forest that called orienteering. Orienteering is a sport where contestants define their own direction among control point or specially defined on map. There is various kind of orienteering, the most common of orienteering is doing by walking. This activity route is outstretched about $2 \mathrm{~km}$ for beginners and children, up to $12 \mathrm{~km}$ for professional.
Various kind of analytics method has been used to solve the orienteering problem. At the beginning, the orienteering problem was solved using exact approach that usually using mathematics model such as Dynamic Programming, Branch and Bound, Binary Integer Programming, and branch and cut to find the best solution as discribed in [1]. With the growing of a problem, when the size of a problem growing too, the computation time is exponentially growing and the method that mentioned before, became unable to be used. Therefore many researchers focused on a heuristics algorithm.

The objective of solving orienteering problem using heuristics method is to find the best solution efficiently. Some applied methods are Greedy Insertion, Sweepbased insertion, Greedy insertion, path improvement, Random insertion, path improvement as described in [1]. Besides heuristics method, some meta-heuristics method also applied on solving orienteering problem. Some of them are Artificial Neural Network by Wang et al. [2], Genetic Algorithm [3], Harmony Search [4], particle Swarm Optimization [5] and ant colony and tabu search as described in [1].

\section{Cross Entropy Method}

Cross entropy (CE) is a quite new approach in optimization and learning algorithm. Pioneered by Reuven Rubi- 
ensten in 1997, CE method has been used widely in many problems, such as combinatorial optimization, continuous optimization, noisy optimization, and rare event simulation [6]. At these problems, cross entropy can find optimal or near optimal solution with a less computational time. The basic idea of the CE method is to transform the original (combinatorial) optimization problem to an associated stochastic optimization problem, and then handling the stochastic problem efficiently by an adaptive sampling algorithm. Through this process one constructs a random sequence of solutions which converges (probabilistically) to the optimal or at least a reasonable solution. Once the associated stochastic optimization is defined, the CE method follows these two phases:

1) Generation of a sample of random data (trajectories, vectors, etc.) according to a specified random mechanism.

2) Update of the parameters of the random mechanism, on the basis of the data, in order to produce a "better" sample in the next iteration.

This procedure provides the general frame. When we are facing a specific problem, like other metaheuristics, we have to modify CE to fit with our problem. Recently, CE had been applied in credit risk assessment problems for commercial banks [7]. Rubinstein used cross entropy to solve combinatorial and rare-event, estimation [8], Derek Magee applied cross entropy on a sequential scheduling approach to combining multiple object classifiers [9], Kroese and Hui used cross entropy in network reliability estimation [10]. CE application has been widely adopted in the case of a difficult combinatorial such as the maximal cut problem, Traveling Salesman Problem (TSP), quadratic assignment problem, various kinds of scheduling problems and buffer allocation problem (BAP) for production lines [6].

\section{Problem Formulation}

Orienteering Problem is one type of TSP with score. Score is a benefit or a satisfaction that gained from visiting the node (city). The objective of an OP is to define the tour that front end the same node, that maximize total score without break over the maximum distance constraint. This problem inspired by outdoor sport that usually played at mountain or forest that called orienteering. Orienteering is a sport which the contestant define their own direction among control point or specially defined on map. There are various kind of an Orienteering, the most common of an orienteering is doing by walking. This activity route outstretched about $2 \mathrm{~km}$ for a beginner and children, and until $12 \mathrm{~km}$ for professional. This time will be used eastern region of China as a case.

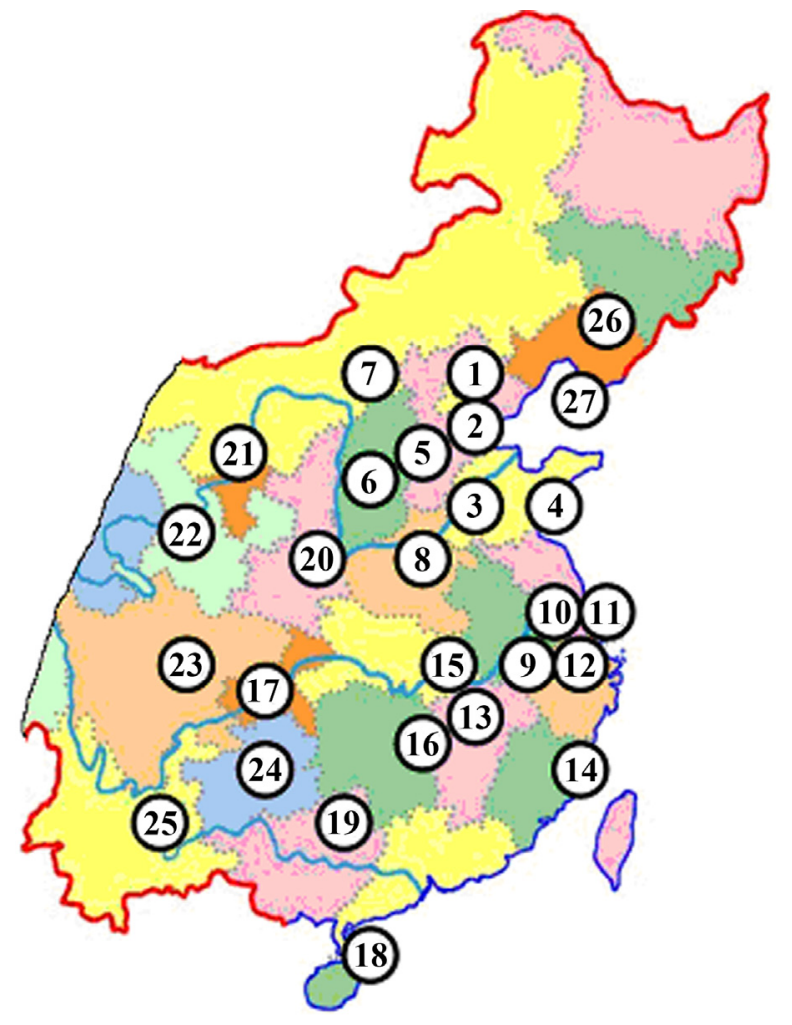

Figure 1. Map of 27 cities in eastern part of China [4].

If a traveler visits eastern part of China, as shown in Figure 1 [4], and he/she wants to travel as many cities as possible with the purpose of best fulfilling multiple factors such as 1) natural beauty, 2) historical interest, 3) cultural event, and 4) business opportunities under the limited total moving distance, his/her travel can become generalized orienteering problem where each city has certain quantified scores for all factors and the estimation of a tour is performed based on the summation of those scores in the tour. The GOP is a generalization of the orienteering problem (OP) and the main difference between the two is that each city in GOP has multiple scores while each city in OP has only one score. In this case, Total distance (DMAX) is set to $5000 \mathrm{~km}$.

The objective function of Generalized Orienteering Problem is maximizing the score of the resulting route as follows.

$$
\operatorname{Max} Z=\sum_{g=1}^{m} W_{g}\left[\left(\sum_{i \in P}\left[S_{g}(i)\right]^{k}\right)^{1 / k}\right]
$$

where $W_{g}$ is the weight of goal $g$, and the exponent $k$ is set to 5 in this problem.

Subject to:

$$
\sum_{j=2}^{n} x_{1 j}=\sum_{i=2}^{n-1} x_{i n}=1
$$




$$
\begin{aligned}
& \sum_{i=2}^{n-1} x_{i k}=\sum_{j=2}^{n-1} x_{k j} \leq 1, k=2, \cdots, n-1 \\
& \sum_{i=1}^{n} \sum_{j=1}^{n} d_{i j} x_{i j} \leq D M A X \\
& x_{i j} \in\{1,0\} \\
& i, j=1, \cdots, n
\end{aligned}
$$

\section{CE Method for GOP}

To solve GOP using CE we propose the following procedure

1) Initialization

Determine $\alpha$ (smoothing parameter), $\rho$ (proportion of elite sample), $\mathrm{N}$ (number of sample), weight (W) of each criteria in the objective function, maximum distance allowed and $p$ (initial ttransition matrix). To generate initial transition matrix with size of $n \times n$, where $n$ is the number of city, use the following formula

$$
\begin{aligned}
& P_{i j}=\sum_{i}^{n} \frac{1}{n}-1, \ldots . \forall i=j \\
& P_{i j}=\frac{1}{n-1}, \ldots \ldots . \forall i \neq j
\end{aligned}
$$

Each cell in the transition matrix, $p(i, j)$, indicates the probability of visiting city $j$ from city $i$. In initial step each city has the same probability to be visited from another city.

\section{2) Route Generation}

Generate $\mathrm{N}$ routes that visit all cities based on the transition matrix obtained in step 1 .

3) Subtour forming

To form subtours, we have to fix the maximum distance allowed. A route is formed by connecting one city with other city and so on until the maximum distance is reached. A city is allowed to be visited once. We use the following distance formula, where the earth assumed to be a sphere:

$$
d(x, y)=r \bullet \arcsin \left(\sin \left(c_{1}\right) \sin \left(\frac{\pi\left(90-b_{1}\right)}{180}\right) / \sin (e)\right)
$$

where

$$
\begin{aligned}
& e=\arctan \left(d_{1}\right)+\arctan \left(d_{2}\right) \\
& d_{1}=\cos \frac{c_{2}}{2} /\left(\cos \frac{c_{3}}{2} \tan \frac{c_{1}}{2}\right), \\
& d_{2}=\sin \frac{c_{2}}{2} /\left(\sin \frac{c_{3}}{2} \tan \frac{c_{1}}{2}\right) \\
& c_{1}=\left(a_{1}-a_{2}\right) \frac{\pi}{180},
\end{aligned}
$$

$$
\begin{aligned}
& c_{2}=\left(b_{2}-b_{1}\right) \frac{\pi}{180}, \\
& c_{3}=\pi-\left(b_{1}+b_{2}\right) \frac{\pi}{180}
\end{aligned}
$$

4) Score Calculation

After we have the routes, we can compute the score of each route by applying Equation (1). The score represents the fitness. In this case we seek to have maximum score.

5) Elite sample selection

After the score of each route is computed, we chose an elite sample with size of $\rho^{*} \mathrm{~N}$ from the whole sample $(\mathrm{N})$. First, we sort the scores from the highest to the lowest. Then, we chose the highest score from this elite sample. Use this elite sample to update the next transition matrix. By this step a route with maximum score will have higher probability than other routes.

6) Parameter updating

After we get the elite sample, we calculate the empirical probability by the following formula:

$$
r_{i, j}=\frac{n_{i, j}}{\rho \times N}
$$

where $n_{i, j}$ denotes how many times city $i$ and $j$ are connected in all routes in the generated sample. We then update the transition matrix using the following formula:

$$
p(i, j)=\alpha \times r+(1-\alpha) \times p_{\text {old }}
$$

where $p_{\text {old }}$ is the transition matrix form previous iteration and $r$ is the empirical probability.

7) Stopping Criteria Checking

If the stopping criteria is met then stop. Otherwise, repeat steps 3 until 7 . One of the stopping criteria commonly used in $\mathrm{CE}$ is the maximum absolute difference between two consequtive transition matrix. If this value is less than $\varepsilon$, then stop.

\section{Illustration}

To explain how this method works, we give an example below.

As example for solving the problem using CE, we use the simple samples, with 5 cities, with 4 scores for each criteria. For weight, we use $\mathrm{W}_{0}=\left(\begin{array}{llll}0.25 & 0.25 & 0.25 & 0.25\end{array}\right)$. The results will be checked by comparing with the enumerative results for validation (Figure 2). There is data shown in Tables $\mathbf{1}$ and $\mathbf{2}$.

Table 1. Distance matrix.

\begin{tabular}{cccccc}
\hline & 1 & 2 & 3 & 4 & 5 \\
\hline 1 & 0.00 & 106.91 & 364.05 & 549.32 & 264.08 \\
2 & 106.91 & 0.00 & 277.33 & 442.44 & 263.55 \\
3 & 364.05 & 277.33 & 0.00 & 305.77 & 269.00 \\
4 & 549.32 & 442.44 & 305.77 & 0.00 & 562.55 \\
5 & 264.08 & 263.55 & 269.00 & 562.55 & 0.00 \\
\hline
\end{tabular}


Table 2. Scores of each node.

\begin{tabular}{cccccc}
\hline No & Nama & S1 & S2 & S3 & S4 \\
\hline 1 & Beijing & 8 & 10 & 10 & 7 \\
2 & Tianjin & 6 & 5 & 8 & 8 \\
3 & Jinan & 7 & 7 & 5 & 6 \\
4 & Qingdao & 7 & 4 & 5 & 7 \\
5 & Shijiazhuang & 5 & 4 & 5 & 5 \\
\hline
\end{tabular}

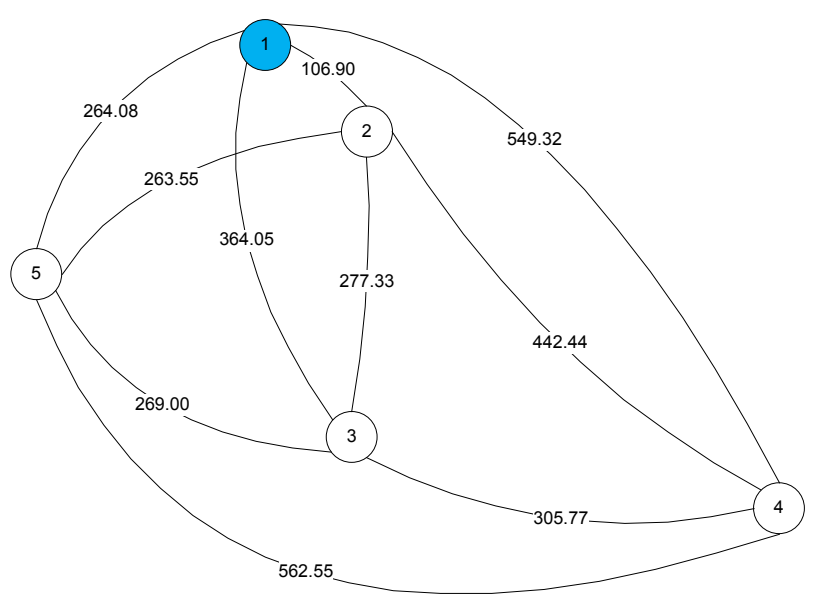

Figure 2. Validation node.

1) Initialization

For initialization let $\alpha$ (smoothing parameter) $=0.8, \rho$ (proportion of elite sample) $=0.3, \mathrm{~N}$ (number of sample every iteration $)=10$, and the weight that used $(0.250 .25$ 0.250 .25 ).

We generate initial transition matrix with size of $n \times n$ where $n$ is the number of city, in this case $n=5$, by using the following formula

$$
\begin{aligned}
& P_{i j}=\sum_{i}^{n} \frac{1}{n}-1, \ldots . \forall i=j \\
& P_{i j}=\frac{1}{n-1}, \ldots . . \forall i \neq j
\end{aligned}
$$

Using this formula we obtain the following transition matrix:

In this phase we generate routes that visit all cities based on the transition matrix in Figure 3. The results are shown in Table 3.

$$
p=\left[\begin{array}{ccccc}
0 & 0.25 & 0.25 & 0.25 & 0.25 \\
0.25 & 0 & 0.25 & 0.25 & 0.25 \\
0.25 & 0.25 & 0 & 0.25 & 0.25 \\
0.25 & 0.25 & 0.25 & 0 & 0.25 \\
0.25 & 0.25 & 0.25 & 0.25 & 0
\end{array}\right]
$$

Figure 3. Transition matrix.
Table 3. Initial routes that generated at iteration 1.

\begin{tabular}{cc}
\hline No & Route \\
\hline 1 & $1-4-5-3-2$ \\
2 & $1-3-4-2-5$ \\
3 & $1-4-5-2-3$ \\
4 & $1-3-2-4-5$ \\
5 & $1-4-5-3-2$ \\
6 & $1-2-5-4-3$ \\
7 & $1-2-4-2-5$ \\
8 & $1-3-5-4-2$ \\
9 & $1-3-24-5$ \\
10 & $1-2-5-3-4$ \\
\hline
\end{tabular}

2) Subtour

To form subtour, we have to fix the maximum distance allowed. A subtour is part of route that cut based on maximum distance, in this example the maximum distance is set to $1000 \mathrm{~km}$. From Table 3 we obtained the resulting subtours in Table 4.

Notice that routes number 1,3 and 5 consist only the fisrt city. This means that the distance to next visited city is more than $1000 \mathrm{~km}$.

3) Score Computation

Applying Equation (1) we obtain scores as shown in Table 5.

Table 4. Resulting subtours.

\begin{tabular}{ccc}
\hline No & Route & Distance $(\mathrm{km})$ \\
\hline 1 & 1 & 0.00 \\
2 & $1-3-1$ & 728.11 \\
3 & 1 & 0.00 \\
4 & $1-3-2-1$ & 748.29 \\
5 & 1 & 0.00 \\
6 & $1-2-5-1$ & 634.54 \\
7 & $1-2-1$ & 213.81 \\
8 & $1-3-5-1$ & 897.14 \\
9 & $1-3-2-1$ & 748.29 \\
10 & $1-2-1$ & 213.81 \\
\hline
\end{tabular}

Table 5. Score of each route.

\begin{tabular}{cc}
\hline No Route & Score \\
\hline 1 & 8.75 \\
2 & 9.16 \\
3 & 8.75 \\
4 & 9.72 \\
5 & 8.75 \\
6 & 9.50 \\
7 & 9.42 \\
8 & 9.25 \\
9 & 9.72 \\
10 & 9.42 \\
\hline
\end{tabular}




$$
p=\left[\begin{array}{ccccc}
0 & 0.3167 & 0.5833 & 0.0500 & 0.0500 \\
0.0500 & 0 & 0.0500 & 0.0500 & 0.3167 \\
0.3167 & 0.5833 & 0 & 0.0500 & 0.0500 \\
0.8500 & 0.0500 & 0.0500 & 0 & 0.0500 \\
0.5633 & 0.0500 & 0.0500 & 0.0500 & 0
\end{array}\right]
$$

4) Elite Sample Selection

Using $\rho=0.3$ we have an alite sample of size 3 out of 10. From the score in Table 5 we choose three best values.

5) Parameter Update

Empirical probability from Elite Sample is shown in Figure 4.

We then update the transition matrix using the following formula:

$$
p(i, j)=\alpha \times r+(1-\alpha) \times p_{\text {old }}
$$

where $p_{\text {old }}$ is (probability matrix from the previous iteration), in this example is $p$ in Figure 3, and $r$ is probability matrix in Figure 4 and Table 6. For example to update $p(1,2)$ with $\alpha=0.8$ the calculation is as follows:

$$
p_{(1,2)}=0.8 \times 0.3333+(1-0.8) \times 0.25=0.3167
$$

The new $p$ is shown in Figure 5.

Table 6. Elite sample.

\begin{tabular}{ccc}
\hline No Rute & Rute & Skor \\
\hline 4 & $1-3-2-1$ & 9.72 \\
9 & $1-3-2-1$ & 9.72 \\
6 & $1-2-5-1$ & 9.50 \\
\hline
\end{tabular}

$$
p=\left[\begin{array}{ccccc}
0 & 0.3333 & 0.6667 & 0 & 0 \\
0 & 0 & 0 & 0 & 0.3333 \\
0.3333 & 0.6667 & 0 & 0 & 0 \\
1 & 0 & 0 & 0 & 0 \\
0.6667 & 0 & 0 & 0 & 0
\end{array}\right]
$$

Figure 4. Empirical probability matrix.

$$
P=\left[\begin{array}{ccccc}
0 & 0.3167 & 0.5833 & 0.0500 & 0.0500 \\
0.0500 & 0 & 0.0500 & 0.0500 & 0.3167 \\
0.3167 & 0.5833 & 0 & 0.0500 & 0.0500 \\
0.8500 & 0.0500 & 0.0500 & 0 & 0.0500 \\
0.5633 & 0.0500 & 0.0500 & 0.0500 & 0
\end{array}\right]
$$

Figure 5. Updated transition matrice.

6) Stopping Criteria Checking

The stopping crieria used in this experiment is the maximum absolute difference between two consequtive transition matrix is less than 0.005 .

7) Optimal Route

After doing steps 1 to 8 , we found the optimal route as shown in Table 7. The table also shows the enumerative result.

Table 7. Result comparison CE-GOP and enumeration.

\begin{tabular}{lccc}
\hline Method & Optimal Route & Distance & Score \\
\hline Enumeration & $1-2-3-5-1$ & 917.3 & 9.79 \\
CE-GOP & $1-2-3-5-1$ & 917.3 & 9.79 \\
\hline
\end{tabular}

Table 8. Comparison between CE, HS, and ANN.

\begin{tabular}{ccccc}
\hline Weight & Method & Distance & Score & Route \\
\hline \multirow{2}{*}{ W0 } & CE & 4993.4 & 12.3793 & $1-2-3-10-11-12-9-13-17-19-16-20-6-5-1$ \\
& HS & 4993.4 & 12.3793 & $1-2-3-10-11-12-9-13-17-19-16-20-6-5-1$ \\
& ANN & 4993.4 & 12.3793 & $1-2-3-10-11-12-9-13-17-19-16-20-6-5-1$ \\
W1 & CE & 4946.0 & 13.1037 & $1-2-3-8-24-19-16-13-9-12-10-4-27-1$ \\
& HS & 4985.4 & 13.0825 & $1-2-3-15-24-19-13-9-12-10-2-27-1$ \\
& ANN & 4987.7 & 13.0529 & $1-2-3-4-10-11-12-9-13-16-19-24-20-6-5-1$ \\
W2 & CE & 4974.4 & 12.5617 & $1-26-27-3-10-12-9-13-15-20-8-6-5-2-1$ \\
& HS & 4910.6 & 12.5617 & $1-26-27-4-10-12-9-13-16-15-20-8-3-2-1$ \\
& ANN & 4875.1 & 12.5070 & $1-2-26-27-3-10-11-12-9-13-15-20-6-5-1$ \\
W3 & CE & 4987.5 & 12.7826 & $1-2-3-5-6-20-8-15-16-13-9-12-11-10-4-27-1$ \\
& HS & 4987.5 & 12.7826 & $1-2-3-5-6-20-8-15-16-13-9-12-11-10-4-27-1$ \\
& ANN & 4987.5 & 12.7826 & $1-2-3-5-6-20-8-15-16-13-9-12-11-10-4-27-1$ \\
W4 & CE & 4956.4 & 12.4273 & $1-2-3-8-15-16-17-14-12-11-10-4-27-1$ \\
& HS & 4845.2 & 12.4009 & $1-2-27-4-10-11-12-14-17-16-15-3-1$ \\
\hline
\end{tabular}




\section{Experiments and Analysis}

We show the results of numerical experiments and the comparison with those of other methods in Table 8 . There are five set of different weight vectors used in the experiments. These weights were applied on three methods. The values of the weight vectors are $W 0=$ $(0.25,0.25,0.25,0.25), W 1=(1,0,0,0), W 2=(0,1,0$, $0), W 3=(0,0,1,0)$, and $W 4=(0,0,0,1) . W 0=(0.25$, $0.25,0.25,0.25)$, means that each criteria has the same weight. By $W 1=(1,0,0,0)$, we means that only natural beauty is considered. The similar meaning is for W2, W3 and W4. The last three weight vectors stress only one goal and ignore the other three. For W0 and W3, we see that all three methods produce the same routes as well as the score. For W1 and W4, CE produced the best result in terms of score.

For W2, CE and HS produced the same results in terms of score with different Distance and routes. As we know, distance is limited by maximum value of $5000 \mathrm{~km}$.

\section{Conclusions}

In this paper we show how Cross Entropy can be applied for solving Generalized Orienteering Problem whose objective is to find the best tour in eastern part of China. The main steps of CE consist of: generate sample, select elite sample with best fitness, update parameters based on the elite sample, generate next better sample using the updated parameters. This procedure can be succesfully translated for GOP. Generally Cross Entropy shows equal or solution compared to those of Harmony Search and Artificial Neural Network.

\section{REFERENCES}

[1] Z. Sevkli, and F. dan Erdogan Sevilgen, "Variable Neighborhood Search for the Orienteering Problem," A. Levi et al., Eds., ISCIS, Springer-Verlag, Berlin, Heidelberg, 2006, pp. 134-143.

[2] Q. Wang, X. Sun, B. L. Golden and J. Jia, "Using Artificial Neural Networks to Solve the Orienteering Problem," Annals of Operations Research, Vol. 61, No. 1, 1995, pp. 111-120.

[3] Tasgetiren and M. Fatih, "A Genetic Algorithm with an Adaptive Penalty Function for Orienteering Problem," Journal of Economic and Social Research, Vol. 4, No. 2, 2000, pp. 1-26.

[4] G. Z. Woo, T. C. Li, D. Park and J. Yong, "Harmony Search for Generalized Orienteering Problem: Best Touring in China," L. Wang, K. Chen and Y. S. Ong, Eds., Springer-Verlag, Berlin Heidelberg, 2005, pp. 741750 .

[5] Z. Sevkli and F. dan E. Sevilgen, "Particle Swarm Optimization for the Orienteering Problem," Proceedings of International Symposium on Innovation in Intelligent Systems and Applications, Istanbul, 2007, pp. 185-190.

[6] R. Rubinstein and D. Kroese, "The Cross-Entropy Method: A Unified Approach to Combinatorial Optimization," Monte-Carlo Simulation, and Machine-Learning, Springer-Verlag, Berlin, Heidelberg, 2004.

[7] H. Zhou, J. Wang and Y. Qiu, "Application of the Cross Entropy Method to the Credit Risk Assessment in an Early Warning System," International Symposiums on Information Processing, 2008.

[8] R. Y. Rubinstein, "Stochastic Minimum Cross-Entropy Method for Combinatorial Optimization and Rare-Event Estimation," Methodology and Computing in Applied Probability, Vol. 7, No. 1, 2005, pp. 5-50.

[9] D. Magee, "A Sequential Scheduling Approach to Combining Multiple Object Classifiers Using Cross-Entropy," T. Windeatt and F. Roli, Eds., Springer-Verlag, Berlin, Heidelberg, 2003, pp. 135-145.

[10] D. P. Kroese and K.-P. Hui, "Applications of the CrossEntropy Method in Reliability," Computational Intelligence in Reliability Engineering, Vol. 40, 2007, pp. $37-$ 82. 